\title{
Coupled oscillators as models of phantom and scalar field cosmologies
}

\author{
Valerio Faraoni \\ Physics Department, University of Northern British Columbia \\ 3333 University Way, Prince George, B.C., Canada V2N $4 Z 9$ \\ emailvvaraoni@unbc.ca
}

\begin{abstract}
We study a toy model for phantom cosmology recently introduced in the literature and consisting of two oscillators, one of which carries negative kinetic energy. The results are compared with the exact phase space picture obtained for similar dynamical systems describing, respectively, a massive canonical scalar field conformally coupled to the spacetime curvature, and a conformally coupled massive phantom. Finally, the dynamical system describing exactly a minimally coupled phantom is studied and compared with the toy model.
\end{abstract}




\section{Introduction}

The idea that the universe underwent an inflationary epoch early in its history [1] has spurred interest in scalar field cosmology. A scalar field is the simplest, although not the unique, way to fuel inflation. In this context a scalar field, which may come in different forms and with a variety of possible self-interaction potentials, is the only or the dominant form of matter in the field equations of the gravitational theory. Scalar fields have also been considered as candidates for dark matter and, more recently, for dark energy or quintessence. The recent discovery that the universe is undergoing an accelerated expansion that started not long ago [2] introduces the need to either modify gravity or postulate a form of dark energy with exotic properties (negative pressure). Scenarios based on modified gravity without extra forms of energy have thus far not been very successful. It is easier to describe the observed acceleration of the universe by using some form of dark energy, and most models in the literature employ a scalar field [3].

There is marginal evidence [4] that not only the universe accelerates but perhaps it even superaccelerates, i.e., that the Hubble parameter $H$ may increase with time, $\dot{H}>0$, which is equivalent to an effective equation of state parameter $w \equiv P / \rho<-1$, where $\rho$ and $P$ are the effective energy density and pressure of the dark energy. If confirmed, superacceleration cannot be explained by a canonical, minimally coupled scalar field [5], but modifications of the theory are required. One of such modifications, considered by many authors [6], consists in a phantom field with negative kinetic energy. Such a field is obtained in S-theory, supergravity and in higher derivative theories [7]. The action for gravity with a phantom field $\phi$ is

$$
S^{(\text {phantom })}=\int d^{4} x \sqrt{-g}\left[\frac{R}{2 \kappa}+\frac{1}{2} g^{a b} \nabla_{a} \phi \nabla_{b} \phi-V(\phi)\right]
$$

where $\kappa=8 \pi G, R$ is the Ricci curvature of spacetime [8] and $V(\phi)$ is the phantom potential. Another possibility to explain superacceleration is to let a scalar field with positive kinetic energy couple nonminimally to the Ricci curvature of spacetime, as described by the action

$$
S^{(n m c)}=\int d^{4} x \sqrt{-g}\left[\left(\frac{1}{2 \kappa}-\frac{\xi}{2} \phi^{2}\right) R-\frac{1}{2} g^{a b} \nabla_{a} \phi \nabla_{b} \phi-V(\phi)\right],
$$

where $\xi$ is a dimensionless coupling constant. The explicit nonminimal coupling to the curvature introduces extra terms in the equations for the scalar field and the cosmological 
metric, allowing for the possibility of superacceleration with $\dot{H}>0$. A more general coupling between the scalar and gravity, such as the one appearing in scalar-tensor and string theories can also generate superacceleration. For example, bouncing universes and pole-like inflation, which are not possible in general relativity with a minimally coupled canonical scalar, are known in these theories [10]. Quantum effects can also provide a mechanism to generate superacceleration from theories that are classically stable (see, e.g., Refs. $[11,12])$.

Apart from the need to explain superacceleration, there are other, much more compelling reasons to consider a nonminimally coupled field. Nonminimal coupling is introduced by quantum corrections to the action of a classical scalar [13], and is even required at the classical level to preserve the Einstein equivalence principle or to avoid causal pathologies [14].

Of particular interest is the case of conformally coupled scalar field theory with $\xi=1 / 6$. In fact, this value of the coupling constant is an infrared fixed point of the renormalization group [15] and therefore we focus on conformally coupled scalars.

Once one accepts the idea of a phantom field with negative kinetic energy, it is natural to also consider a nonminimally coupled phantom field [16]. While models of nonminimally coupled scalars have been studied since the early days of inflationary theory, the introduction of phantom fields in cosmology is very recent. The defining feature of a phantom, its negative kinetic energy, has been associated with instability and runaway solutions. The possibility of negative energies is a well known feature of scalar-tensor and generalized gravity and seems to be accepted in this context, at least when gravity is classical [10]. Hence, it is not clear that a negative energy is a priori a problem. Moreover, when a field is coupled to gravity the gravitational energy plays a role in the dynamics and no meaningful prescription is available for the energy density of the gravitational field even in general relativity. Issues of principle with negative energy are discussed in section 6. Satisfactory answers to the questions raised, however, come from the understanding of the dynamics, which constitutes the main part of this paper.

The problem of the stability of a phantom has motivated the consideration of a toy model consisting of two coupled oscillators, one with positive and one with negative definite kinetic energy, which exhange energy during motion [17]. The canonical oscillator with positive kinetic energy is supposed to mimic the gravitational field while the second oscillator mimics the phantom field. This dynamical system is also an exact description of the dynamics of a massive conformally coupled scalar field in a FLRW universe, using suitably rescaled variables and conformal time. Section 2 summarizes the equations and motivations for the coupled oscillator model, while section 3 analyzes the dynamics of this system. Section 4 considers a similar system of coupled oscillators - both with pos- 
itive kinetic energy - which describe exactly a conformally coupled phantom in rescaled variables and conformal time. Having learned a lesson from section 4, we can approach in section 5 the exact description of a minimally coupled phantom, without resorting to toy models - the phase space is analyzed in rescaled variables also for this case. Section 6 contains a discussion and the conclusions.

\section{Coupled oscillators as cosmological models}

In this section we summarize the equations of phantom and scalar field cosmology and we show how they can be reduced to a model with two coupled oscillators. We set our analysis in the context of a spatially flat Friedmann-Robertson-Walker universe described by the line element

$$
d s^{2}=-d t^{2}+a^{2}(t)\left(d x^{2}+d y^{2}+d z^{2}\right)
$$

in comoving coordinates $(t, x, y, z)$. In this homogeneous and isotropic universe the scalar field $\phi(t)$ only depends on the comoving time and self-interacts through the potential $V(\phi)$. For simplicity it is assumed that the scalar field is the only form of matter present.

\subsection{Phantom cosmologies}

A minimally coupled phantom field in the metric (2.1) has energy density and pressure

$$
\begin{aligned}
& \rho_{p}=-\frac{1}{2} \dot{\phi}^{2}+V(\phi), \\
& P_{p}=-\frac{1}{2} \dot{\phi}^{2}-V(\phi),
\end{aligned}
$$

which correspond to the usual expressions for the energy density and pressure of a minimally coupled scalar but with the sign of the kinetic energy inverted. A positive potential $V(\phi)$ is necessary if the energy density $\rho_{p}$ is to be non-negative. Although this requirement is sometimes dropped [17] most relativists are reluctant to give up the weak energy condition, while all the other energy conditions are progressively being abandoned [18].

The pressure $P_{p}$ in eq. (2.3) is more negative than for a canonical scalar field, thus making it possible to achieve a superquintessence phase with $P_{p}<-\rho_{p}$. A minimally coupled scalar with canonical kinetic energy density $+\dot{\phi}^{2} / 2$ in Einstein gravity cannot achieve $P<-\rho$ [5]. 
The Einstein field equations in the metric (2.1) and with the phantom $\phi$ as material source are

$$
\begin{gathered}
H^{2}=\frac{\kappa}{6}\left[-\dot{\phi}^{2}+2 V(\phi)\right], \\
\dot{H}+H^{2}=\frac{\kappa}{3}\left[\dot{\phi}^{2}+V(\phi)\right], \\
\ddot{\phi}+3 H \dot{\phi}-\frac{d V}{d \phi}=0,
\end{gathered}
$$

where an overdot denotes differentiation with respect to the comoving time $t$. Only two equations in the set (2.4)-(2.6) are independent - when $\dot{\phi} \neq 0$ one can derive the Klein-Gordon equation (2.6) from the other two, or from the conservation equation $\dot{\rho}_{p}+3 H\left(P_{p}+\rho_{p}\right)=0$ satisfied by the phantom.

The field equations (2.4)-(2.6) can be derived from the Lagrangian

$$
L_{0}=3 a \dot{a}^{2}+\kappa a^{3}\left[\frac{\dot{\phi}^{2}}{2}+V(\phi)\right],
$$

or from the Hamiltonian

$$
\mathcal{H}_{0}=a^{3}\left[H^{2}+\frac{\kappa}{6} \dot{\phi}^{2}-\frac{\kappa V(\phi)}{3}\right]
$$

A toy model for phantom cosmology consists of two coupled oscillators, one with positive-definite and one with negative-definite kinetic energy, described by the Lagrangian

$$
L_{T}=\frac{\dot{x}^{2}}{2}-\frac{\dot{y}^{2}}{2}-\frac{m_{x}^{2}}{2} x^{2}-\frac{m_{y}^{2}}{2} y^{2}-\frac{\mu^{2}}{2} x^{2} y^{2},
$$

or by the associated Hamiltonian

$$
\mathcal{H}_{T}=\frac{\dot{x}^{2}}{2}-\frac{\dot{y}^{2}}{2}+\frac{m_{x}^{2}}{2} x^{2}+\frac{m_{y}^{2}}{2} y^{2}+\frac{\mu^{2}}{2} x^{2} y^{2} .
$$

A similar model was considered in Ref. [17]. However, these authors choose the opposite sign for the term $m_{y}^{2} y^{2} / 2$, and the energy of the phantom turns out to be $\rho_{p}=-\left(\dot{\phi}^{2}+m_{\phi}^{2} \phi^{2}\right) / 2$ and negative-definite instead of $\rho_{p}=\left(-\dot{\phi}^{2}+m_{\phi}^{2} \phi^{2}\right) / 2$. While in the toy model the negative kinetic and potential energy of the "phantom" oscillator $y$ can be offset by the positive interaction energy $\mu^{2} x^{2} y^{2} / 2$, this is not a possibility for the real phantom field. In the exact phantom model, expressions for the interaction 
energy density between gravitational and phantom fields and for the gravitational field energy density are not available, and it is desirable to keep the energy of the phantom positive and preserve the weak energy condition. This explains the difference between our Lagrangian (2.9) and that of Ref. [17].

As remarked in Ref. [17], while the total energy of the system is constant, the energy of the phantom oscillator could decrease ad infinitum and the energy of the other oscillator could increase without bound - the system could not have a stable ground state. This argument has been presented before in the context of scalar-tensor gravity, which also has negative energies [10].

The Euler-Lagrange equations derived from the Lagrangian (2.9) are

$$
\begin{aligned}
& \ddot{x}+\left(m_{x}^{2}+\mu^{2} y^{2}\right) x=0, \\
& \ddot{y}-\left(m_{y}^{2}+\mu^{2} x^{2}\right) y=0 .
\end{aligned}
$$

The physically interesting case corresponds to a massless graviton and $m_{x}=0$, while the phantom field is very light with a mass $m_{\phi} \approx 10^{-33} \mathrm{eV}$. Eq. (2.11) resembles an harmonic oscillator equation with a real $y$-dependent effective mass, while eq. (2.12) exhibits a purely imaginary effective mass $i \sqrt{m_{y}^{2}+\mu^{2} x^{2}}$. The first property is associated with stability and the second one with instability. By changing the sign of the mass term for the phantom, as done in Ref. [17], one obtains an effective mass squared $\left(m_{y}^{2}-\mu^{2} x^{2}\right)$ for the $y$-oscillator, which is positive and corresponds to stability if $\mu^{2} x^{2}<m_{y}^{2}$. Since the phantom mass is constrained to be extremely small, one would have to fine-tune the coupling parameter $\mu^{2}$ to achieve stability [17].

In the next section we consider the toy model for a massless phantom obtained by setting $m_{x}=m_{y}=0$ and $\mu^{2}=1$ and described by the dynamical system

$$
\begin{aligned}
& \ddot{x}=-x y^{2}, \\
& \ddot{y}=x^{2} y,
\end{aligned}
$$

which is invariant under the transformations $(x, y) \rightarrow(-x, y)$ and $(x, y) \rightarrow(x,-y)$.

\subsection{Conformally coupled scalar field cosmology}

There are many reasons to believe that a scalar field in a curved space couples nonminimally to the Ricci curvature: nonminimal coupling is induced by quantum corrections to the classical action [13] and is required to renormalize the scalar field theory [19]. In 
particular, conformal coupling is an infrared fixed point of the renormalization group [15] and is required by the Einstein equivalence principle and to avoid acausal propagation of $\phi$-waves [14]. Hence, we consider a conformally coupled massive scalar field. The equations of motion obtained by varying the action (1.2) with $\xi=1 / 6$ and $V=m^{2} \phi^{2} / 2$ are $([20,21],[23]-[29])$

$$
\begin{gathered}
\dot{H}+2 H^{2}-\frac{\kappa m^{2}}{6} \phi^{2}=0, \\
\frac{\kappa}{2} \dot{\phi}^{2}+\kappa H \phi \dot{\phi}-3 H^{2}\left(1-\frac{\kappa}{6} \phi^{2}\right)+\frac{\kappa m^{2}}{2} \phi^{2}=0, \\
\ddot{\phi}+3 H \dot{\phi}+\frac{R}{6} \phi+m^{2} \phi=0,
\end{gathered}
$$

and they incorporate the Hamiltonian constraint

$$
H^{2}=\frac{\kappa}{3} \rho .
$$

The effective energy density and pressure of the scalar can be identified in three physically inequivalent ways [22] - only one of these guarantees conservation of energy, and it yields

$$
\begin{gathered}
\rho=\frac{\dot{\phi}^{2}}{2}+\frac{m^{2}}{2} \phi^{2}+\frac{1}{2} H \phi(H \phi+2 \dot{\phi}), \\
P=\frac{\dot{\phi}^{2}}{2}-\frac{m^{2}}{2} \phi^{2}-\frac{1}{6}\left[4 H \phi \dot{\phi}+2 \dot{\phi}^{2}+2 \phi \ddot{\phi}+\left(2 \dot{H}+3 H^{2}\right) \phi^{2}\right] .
\end{gathered}
$$

The expression (2.19) of $\rho$ is considerably complicated but the problem can be reduced to a system of two oscillators with sharply defined energies in a fictitious Minkowski space as follows. By using the rescaled variables [23]-[25]

$$
x \equiv m a, \quad y \equiv \sqrt{\frac{\kappa m^{2}}{6}} a \phi
$$

and the conformal time $\eta$ defined by $d t=a d \eta$, the equations of motion become

$$
\begin{aligned}
& x^{\prime \prime}=x y^{2}, \\
& y^{\prime \prime}=-x^{2} y,
\end{aligned}
$$

where a prime denotes differentiation with respect to $\eta$. Eqs. (2.22) and (2.23) are invariant under the transformations $(x, y) \rightarrow(-x, y)$ and $(x, y) \rightarrow(x,-y)$ and they can be derived from the Lagrangian

$$
L_{1}=\frac{\left(y^{\prime}\right)^{2}}{2}-\frac{\left(x^{\prime}\right)^{2}}{2}-\frac{1}{2} x^{2} y^{2} .
$$


The associated Hamiltonian is

$$
\mathcal{H}_{1}=\frac{\left(y^{\prime}\right)^{2}}{2}-\frac{\left(x^{\prime}\right)^{2}}{2}+\frac{1}{2} x^{2} y^{2}
$$

and the Hamiltonian constraint is $\mathcal{H}_{1}=0$. Note that it is incorrect to think of $x$ and $y$ as describing gravity and matter, respectively, because $y$ is a mixture of the gravitational and scalar field degrees of freedom. However, $x=m a$ is associated with gravity and it is its effective kinetic energy that is negative here.

Formally, the system (2.22) and (2.23) can be obtained from the system (2.13) and (2.14) by the exchange of variables $(x, y) \rightarrow(y, x)$ and $t \rightarrow \eta$. Note however that the variables $x$ and $y$ and the time coordinates $t$ and $\eta$ used in the two cases have different physical meanings. Moreover, for the toy model (2.13) and (2.14) we allow any value of $x$, while in conformal scalar field cosmology the restriction $x>0$ applies.

\section{Oscillator model for massless phantom and con- formal massive field}

The system of coupled oscillators (2.13) and (2.14) was studied by Castagnino et al. [25]. Their results are:

- The fixed points of the system are $\left(x_{0}, 0\right)$ and $\left(0, y_{0}\right)$ and they are all unstable. The system of two oscillators does not have stable positions of equilibrium.

- All the orbits in the phase space (apart, of course, from the fixed points) go to infinity as $t \rightarrow+\infty$ with $y(t) \rightarrow+\infty$ monotonically while $x(t) \rightarrow 0$ oscillating.

- There are no cycles and there is no chaos.

- An asymptotic solution that seems to be generic is

$$
x(t) \approx \frac{2}{t} \sin \left(\frac{t^{3}}{3}\right), \quad y(t) \approx t^{2} .
$$

The kinetic energy associated with the $x$-oscillator is

$$
K^{(x)}=\frac{(\dot{x})^{2}}{2} \approx 2 t^{2} \cos ^{2}\left(\frac{t^{3}}{3}\right)
$$


and oscillates with divergent amplitude, while the kinetic energy of the $y$-oscillator, $K^{(y)}=-(\dot{y})^{2} / 2 \approx-2 t^{2} \rightarrow-\infty$. This corresponds to the "instability" described by Carroll et al. However, Castagnino et al. argue on the basis of their numerical analysis that this solution is an attractor, i.e., it is dynamically stable.

Technically, while proving that $x(t) \rightarrow 0$ as $t \rightarrow+\infty$, Castagnino et al. do not prove that $x(t)$ oscillates while going to zero. However, this is straightforward to demonstrate. Assume that $x \rightarrow 0$ monotonically. Then, if $x>0$ the curve representing $x(t)$ has the $t$-axis as horizontal asymptote and $\dot{x}<0, \ddot{x}>0$. This contradicts eq. (2.13) which guarantees that $\ddot{x}=-x y^{2}<0$. The same reasoning applies if $x<0$ goes to zero monotonically. Hence, $x(t)$ must oscillate.

Let us turn now to the case of a massive conformally coupled scalar field. Castagnino et al. actually studied the dynamical system (2.13) and (2.14) but their variables are not defined as in our eqs. (2.21) $-x$ and $y$ are exchanged in their definition, so their system (2.13) and (2.14) is physically equivalent to our system (2.22) and (2.23) and their results are derived using conformal time [30]. The proofs in Ref. [25] are then given by considering the limit $\eta \rightarrow+\infty$. This does not necessarily correspond to large comoving times, which is what is instead desired. For example, in a de Sitter space the scale factor is $a=a_{0} \mathrm{e}^{H t}=-\frac{1}{H \eta}$ and $t \rightarrow+\infty$ corresponds to $\eta \rightarrow 0^{-}$. Moreover, if big rip solutions of the kind $a=a_{0}\left(t_{0}-t\right)^{-1}$ are present, both comoving and conformal time stop at a finite value. However, one can consider the least upper bound $\eta_{*}$ on the possible values of $\eta$ along the orbits of the solutions and replace the limit $\eta \rightarrow+\infty$ with $\eta \rightarrow+\eta_{*}$ in the proofs of Ref. [25].

When translated in terms of physical variables $a$ and $\phi$ and in comoving time $t$ and by imposing the restriction $x>0$, the interpretation of their results is as follows.

- The fixed points of the system $\left(x_{0}, 0\right)$ are Minkowski spaces and they are all unstable. There are no attractor points.

- All the orbits in the phase space (apart, of course, from the fixed points) go to infinity as $t \rightarrow+\infty$ with $y(t) \rightarrow+\infty$ monotonically while $x(t) \rightarrow 0$ oscillating. These solutions correspond to universes that expand to infinity with scalar field that goes to zero oscillating. This result is consistent with the known phenomenology of a nonminimally coupled scalar field: oscillating solutions for $\xi>0$ (e.g., [26]) and non-oscillating ones for $\xi<0$ (e.g., [27]).

- There are no cycles and there is no chaos. 
- The asymptotic solution

$$
x(t) \approx \frac{2}{\eta} \sin \left(\frac{\eta^{3}}{3}\right), \quad y(t) \approx \eta^{2}
$$

corresponds to a matter-dominated universe with $a(t)=a_{0} t^{2 / 3}$.

An analysis on the lines of that of Ref. [16] leads to the conclusion that the dynamical system is not integrable for general values of the parameter $\mu$ : however, it is not chaotic. (If a quartic self-interaction for the scalar and a cosmological constant are added to the scenario, there are special values of the parameters for which the system is integrable $[28,24]$ - however, these cases are fine-tuned and not physically relevant.)

\section{Conformally coupled phantom}

Once one accepts the use of a phantom field, it is natural to consider a nonminimally coupled phantom [16]. Here we focus on a massive conformally coupled phantom field for which the Klein-Gordon equation is

$$
\ddot{\phi}+3 H \dot{\phi}-m^{2} \phi-\xi R \phi=0 .
$$

In terms of the rescaled variables $x$ and $y$ of eq. (2.21) and of conformal time $\eta$, the field equations can be derived from the Lagrangian

$$
L_{2}=\frac{\left(x^{\prime}\right)^{2}}{2}+\frac{\left(y^{\prime}\right)^{2}}{2}+\frac{1}{2} x^{2} y^{2}
$$

or from the Hamiltonian

$$
\mathcal{H}_{2}=\frac{\left(x^{\prime}\right)^{2}}{2}+\frac{\left(y^{\prime}\right)^{2}}{2}-\frac{1}{2} x^{2} y^{2} .
$$

The Lagrangian (4.2) is actually equivalent (apart from an irrelevant overall sign) to the Lagrangian $L_{1}$ of eq. (2.24) in which the conformally coupled scalar is turned into a phantom, i.e., in which both gravity and the rescaled phantom have negative kinetic energies.

The dynamical system is [16]

$$
\begin{aligned}
& x^{\prime \prime}=x y^{2}, \\
& y^{\prime \prime}=x^{2} y,
\end{aligned}
$$


and the energy constraint is $\mathcal{H}_{2}=0$. The dynamical system (4.4) and (4.5) is invariant under any of the exchanges $(x, y) \rightarrow(y, x),(x, y) \rightarrow(-x, y)$, or $(x, y) \rightarrow(x,-y)$ and the phase space is 3-dimensional in these variables. The fixed points are the empty Minkowski spaces $\left(x_{0}, 0\right)$. A series of results analogous to those seen in the previous section hold, and they are proven in the same way.

Theorem all the (nonstationary) orbits in the phase space go to infinity with $x \rightarrow+\infty$.

Theorem there are no closed orbits in the phase space.

Again, all the solutions that are not stationary represent universes expanding to infinity, all the Minkowski fixed points are unstable, and there is no chaos. Of course, the symmetry between $x$ and $y$ leads to features that are different from the previous case:

Theorem both variables $x$ and $y$ diverge without oscillations as $\eta \rightarrow \eta_{*}$.

The proof is as follows. Consider the function $I\left(x, x^{\prime}\right) \equiv x^{\prime} / x$, which is positive along orbits different from the fixed points. By differentiating $I$ along the orbits and using eq. (4.4) one obtains

$$
\frac{d I}{d \eta}=\frac{x^{2} y^{2}-\left(x^{\prime}\right)^{2}}{x^{2}} .
$$

The energy constraint $\mathcal{H}_{2}=0$ yields

$$
\frac{d I}{d \eta}=\left(\frac{y^{\prime}}{x}\right)^{2}
$$

which is strictly positive along the orbits of non-static solutions. $I$ is positive and strictly

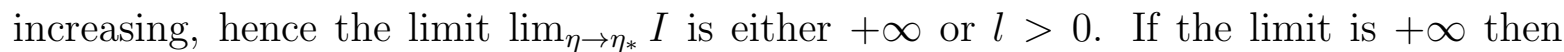
$\frac{x^{\prime}}{x} \rightarrow+\infty$ implies that $x^{\prime} \rightarrow+\infty$ faster than $x$, and also $x^{\prime \prime} \rightarrow+\infty$. The energy constraint implies that

$$
y^{2}=\left(\frac{x^{\prime}}{x}\right)^{2}+\left(\frac{y^{\prime}}{x}\right)^{2} \geq\left(\frac{x^{\prime}}{x}\right)^{2}
$$

diverges. Since $y^{2} \rightarrow+\infty, y$ cannot oscillate and have zeros. Hence we have one of the two possibilities $y \rightarrow \pm \infty$. The second derivative $y^{\prime \prime}=x^{2} y$ has the sign of $y$ and is either positive or negative, but it does not change sign. Hence $y$ does not oscillate. 
If instead the limit of $I$ is finite and there is a horizontal asymptote, then $I^{\prime}=$ $\left(y^{\prime} / x\right)^{2} \rightarrow 0^{+}$. Since $I^{\prime} \geq 0$ and $I^{\prime} \rightarrow 0$, it is $I^{\prime \prime} \rightarrow 0^{+}$. Now,

$$
\frac{d^{2} I}{d \eta^{2}}=\frac{2 y^{\prime}}{x^{3}}\left(x y^{\prime \prime}-x^{\prime} y^{\prime}\right)=2\left[y y^{\prime}-\frac{x^{\prime}}{x}\left(\frac{y^{\prime}}{x}\right)^{2}\right] \rightarrow 0^{+}
$$

and, by taking the limit of this quantity as $\eta \rightarrow \eta_{*}$ and using the fact that $x^{\prime} / x \rightarrow l$ and $y^{\prime} / x \rightarrow 0$, one obtains

$$
\lim _{\eta \rightarrow \eta_{*}} y y^{\prime}=0^{+} .
$$

Now we have $I^{\prime \prime}>0$, or $y y^{\prime}>I\left(\frac{y^{\prime}}{x}\right)^{2}>0$. Either $y>0$ and $y^{\prime}>0$ or $y<0$ in conjunction with $y^{\prime}<0$. In the first case, in order for $y$ to go to zero, $y$ must decrease and it must be $y^{\prime}<0$ : we then have a contradiction. The same reasoning applies to the case $y<0$ with $y^{\prime}<0$, reaching again a contradiction. Hence the limit of $I$ cannot be finite. This completes the proof.

The system is integrable if $\mu=0$, which describes the trivial case of two free noninteracting particles. In general, however, the system is not Liouville-integrable and in this sense it is dynamically complex [16], but it is not chaotic.

Finally, we present the two exact solutions

$$
\begin{gathered}
x_{1}(\eta)=y_{1}(\eta)=\frac{ \pm \sqrt{2}}{\eta-\eta_{0}}, \\
x_{2}(\eta)=-y_{2}(\eta)=\frac{\mp \sqrt{2}}{\eta-\eta_{0}},
\end{gathered}
$$

where $\eta_{0}$ is a constant and the sign is chosen so that $x>0$. The relation $d t=a d \eta$ is easily integrated to express these solutions in terms of comoving time $t$ and see that they represent the expanding or contracting de Sitter spaces

$$
a(t)=a_{0} \mathrm{e}^{ \pm \frac{m t}{\sqrt{2}}}, \quad \phi(t)= \pm \sqrt{\frac{6}{\kappa}} .
$$

This special value of the scalar $\phi$ corresponds to a divergent effective gravitational coupling $\kappa_{e f f} \equiv \kappa\left(1-\kappa \phi^{2} / 6\right)^{-1}$ and to a non-removable curvature singularity in Bianchi I universes $[29,33]$. 


\section{Minimally coupled phantom in rescaled variables}

We can now approach exactly the problem of the minimally coupled massive phantom without resorting to toy models by using the rescaled variables (2.21) and compare the equations of motion with those obtained in the previous sections. In terms of the variables $x$ and $y$, the equations of motion are

$$
\begin{aligned}
& x^{\prime \prime}=-\frac{\left(x^{\prime}\right)^{2}}{x}+3 x y^{2}, \\
& y^{\prime \prime}=-\frac{\left(x^{\prime}\right)^{2} y}{x^{2}}+3 y^{3}+x^{2} y,
\end{aligned}
$$

while the energy constraint is

$$
\left(x^{\prime}\right)^{2}+\left(y^{\prime}-\frac{y x^{\prime}}{x}\right)^{2}=x^{2} y^{2},
$$

and the (rescaled) effective Lagrangian and Hamiltonian (2.7) and (2.8) are

$$
\begin{gathered}
L_{0}=\frac{1}{x}\left[\left(x^{\prime}\right)^{2}+x^{2} y^{2}+\left(y^{\prime}-\frac{x^{\prime} y}{x}\right)^{2}\right], \\
\mathcal{H}_{0}=\frac{\left(x^{\prime}\right)^{2}}{x}+\frac{1}{x}\left(y^{\prime}-\frac{y x^{\prime}}{x}\right)^{2}-x y^{2} .
\end{gathered}
$$

Eqs. (5.1) and (5.2) are rather complicated in comparison to the cases studied in section 3 and 4 , due to the fact that now the phantom is not conformally coupled.

The fixed points are again Minkowski spaces $\left(x_{0}, 0\right)$. In the new variables we are able to derive various results.

Theorem all the orbits in the phase space (apart from the stationary points) go to infinity.

To prove this statement consider the function $F\left(x, x^{\prime}\right)=x x^{\prime}$. By differentiating along the orbits of the solutions and using eq. (5.1) one obtains $F^{\prime}=3 x^{2} y^{2}>0$ on all the non-static orbits. Since $F(\eta)$ is positive and monotonically increasing it is either $\lim _{\eta \rightarrow \eta_{*}} F(\eta)=C>0$ or $\lim _{\eta \rightarrow \eta_{*}} F(\eta)=+\infty$. We show that $F$ cannot tend to a finite limit. In fact, if $F(\eta) \rightarrow C$, the asymptotic equation $x x^{\prime} \approx C$ is satisfied and the 
asymptotic solution is $x=\sqrt{2 C \eta+D}$, where $D$ is an integration constant. Then

$$
x^{\prime \prime} \approx-\frac{C^{2}}{(2 C \eta+D)^{3 / 2}}<0,
$$

while eq. (5.1) combined with the energy constraint yields

$$
x^{\prime \prime}=\frac{1}{x}\left(y^{\prime}-\frac{y x^{\prime}}{x}\right)^{2}+2 x y^{2}>0,
$$

in contradiction with eq. (5.6). Hence $F=x x^{\prime} \rightarrow+\infty$, which implies that $x \rightarrow+\infty$.

Theorem there are no closed orbits (cycles) in phase space.

The proof in Ref. [25] applies also to this case. Hence all the solutions (apart from the fixed points) represent universes expanding to infinity, all the Minkowski space fixed points are unstable, and there is no chaos. Furthermore,

Theorem the variable $y$ diverges without oscillating.

To prove this statement, consider $J\left(x, x^{\prime}\right) \equiv x^{\prime} / x$, which is well defined for $\eta \rightarrow \eta_{*}$ and is positive because $F \equiv x x^{\prime}>0$ and $x>0$. By differentiating along the orbits and using eq. (5.1) one obtains

$$
J^{\prime}(\eta)=-2\left(\frac{x^{\prime}}{x}\right)^{2}+3 y^{2} .
$$

The energy constraint (5.3) then yields

$$
J^{\prime}(\eta)=\frac{2}{x^{2}}\left(y^{\prime}-\frac{y x^{\prime}}{x}\right)^{2}+y^{2}>0 .
$$

Hence $J$ is positive and increasing and the limit of $J$ is either finite, $\lim _{\eta \rightarrow \eta_{*}} J=k>0$, or plus infinity. The limit cannot be finite, or else there is a horizontal asymptote of $J$ and $J^{\prime} \rightarrow 0$, which implies that both $\frac{1}{x^{2}}\left(y^{\prime}-\frac{y x^{\prime}}{x}\right)^{2} \rightarrow 0$ and $y \rightarrow 0$. But then the energy constraint (5.3) implies that

$$
\left(\frac{x^{\prime}}{x}\right)^{2}=-\frac{1}{x^{2}}\left(y^{\prime}-\frac{y x^{\prime}}{x}\right)^{2}+y^{2} \rightarrow 0 .
$$


On the other hand, if $J \rightarrow k$, it is asymptotically $x^{\prime} / x \approx k>0$, in contradiction with $x^{\prime} / x \rightarrow 0$.

Hence, it must be $J=x^{\prime} / x \rightarrow+\infty$, which implies that $x^{\prime} \rightarrow+\infty$ and, using again eq. (5.3),

$$
y^{2}=\left(\frac{x^{\prime}}{x}\right)^{2}+\frac{1}{x^{2}}\left(y^{\prime}-\frac{y x^{\prime}}{x}\right)^{2} \geq J^{2} \rightarrow+\infty
$$

and $y \rightarrow \pm \infty$. Moreover, $y$ cannot oscillate or it would go through zeros, which is in contradiction with $y^{2} \rightarrow+\infty$.

Again, one does not expect the system to be integrable, but there is no chaos.

\section{Discussion and conclusions}

We are now ready to summarize and discuss our findings and to compare them with previous works.

The dynamical system (2.13) and (2.14) that constitutes a toy model for a minimally coupled massless phantom field and a massless graviton in physical time was studied by Castagnino et al. [25]. Their intent was to study the dynamical system describing the physically different situation of a conformally coupled massive scalar in rescaled variables and conformal time. The latter is obtained from the system (2.13) and (2.14) by the exchange $(x, y) \rightarrow(y, x)$ and $t \rightarrow \eta$. The variables $x$ and $y$ and the conformal time employed in Ref. [25] have a physical meaning that is quite different from that of the coupled oscillators in the toy model for the phantom. Carroll et al. [17] considered the toy model for a phantom but added a negative mass term to the potential in order to stabilize the perturbations of the model - the system studied by Castagnino et al. corresponds to the special case of that considered by Carroll et al. in which the phantom is massless and the perturbations are always unstable.

The phase space picture of the dynamical system (2.22) and (2.23) describing a conformally coupled massive scalar field with canonical kinetic energy density is the following [25]. All the fixed points are empty Minkowski spaces and they are unstable. There are no cycles and all the orbits of the solutions (except, of course, the fixed points) represent universes expanding to infinity, while the scalar field goes to zero oscillating. Hence, the matter content (the scalar) tends to infinite dilution in the future while the universe expands. This is consistent with the known phenomenology of nonminimally coupled scalar fields.

The phase space of the dynamical system (4.4) and (4.5) describing a conformally coupled massive phantom field is qualitatively different: there are again unstable fixed 
points representing Minkowski spaces and all the other orbits go to infinity, but the $y$-variable diverges without oscillating. Again, there are no cycles.

We have studied also the exact phase space of a massive, minimally coupled phantom in the rescaled $x$ - and $y$-variables and using conformal time, without resorting to toy models. In these variables, the Lagrangian and Hamiltonian are considerably more complicated than in the previous cases, due to the fact that the $x$ - and $y$-variables are suited for conformal coupling. Nevertheless, by using these variables we can compare our results for a minimally coupled massive phantom with those for conformally coupled canonical scalars and phantoms. The results for this case are as follows. There are again Minkowski space fixed points, which are unstable. All the other orbits go to infinity with $y$ diverging without oscillations, and there are no cycles. Allowing the phantom to be massive does not constrain the orbits to a finite region of the phase space. In fact the phantom "falls up" in the potential $m^{2} \phi^{2} / 2$, which does not have a maximum in which $\phi$ can settle.

There is a difference in the qualitative behaviour of the solutions of the exact equations for the phantom in this case and those of the toy model discussed in Refs. [17, 25]. While in the exact case studied here $x$ and $y$ diverge without oscillating, the toy model has $x \rightarrow 0$ with oscillating behaviour while $y \rightarrow+\infty$. The toy model is clearly inadequate and its solutions are wildly different from the solutions of the equations describing the actual physical system comprising the gravitational field and the phantom.

Finally, let us comment on the instability associated with negative energy which motivated the introduction of a toy model in the first place [17]. The argument that a negative kinetic energy leads to instability is recurrent in Brans-Dicke, scalar-tensor and non-linear gravity theories and many authors regard it as a rationale to discard the Jordan frame formulation of scalar-tensor theories in favour of their Einstein frame counterpart (see Ref. [31] for a review and Ref. [10] for a discussion). This point of view is legitimate in flat space physics but is not compelling when gravity is included in the picture. First, one cannot write expressions for the energy density of the gravitational field (corresponding to the $x$-oscillator in the toy model) and the interaction energy density between the two fields (corresponding to the $\mu^{2} x^{2} y^{2} / 2$ term), and it is not clear how such a separation could be achieved. This fact causes ambiguities in the definition of energy density for a scalar that couples nonminimally to the curvature through an explicit non-minimal coupling term or a Brans-Dicke-like term in the action [22, 10]. Indeed, one can associate unambiguously an energy to scalar waves in linearized BransDicke gravity, and this energy turns out to be negative - hence one would expect an instability. However, the Minkowski background is stable [32]. Second, the discussion of runaway solutions associated with a negative energy instability becomes delicate in the 
cosmological context. A runaway solution representing a universe that expands forever or recollapses is undistinguishable from an "ordinary" cosmological solution with the same properties, which is regarded as perfectly acceptable. It is true that negative energies in scalar-tensor gravity lead to trouble with the formulation of the Cauchy problem or with the quantization of the linearized gravitational field [10], but the negative energy cosmological solutions seem acceptable at the classical level.

When the phantom is treated as a quantum field, the instability becomes more worrisome because, in general, a quantum field has many more states than a particle and there are many more channels for decay - this plays a role in how an instability manifests itself. A quantum phantom field can decay into ordinary particles and other phantoms and, vice-versa, particles can decay into phantoms and other particles. Since the direct coupling of the phantom to ordinary matter must be suppressed to a high degree in order not to violate the equivalence principle, there remains the possibility of decays of phantoms into gravitons and vice-versa. Since the classical phantom coupled to the gravitational field would seem to produce negative energy scalars and positive energy gravitons, and gravity couples to the stress-energy tensor of the phantom, the decay process would be ultra-fast without a cutoff. Carroll et al. [17] study this possibility and conclude that it is not unreasonable to obtain lifetimes larger than the age of the universe if a momentum cutoff as low as $10^{-3} \mathrm{eV}$ can be motivated. At present, there is no justification for such a cutoff, while a natural cutoff would instead be at the Planck scale. Hence, although at the classical level the purported instability of the classical unperturbed model does not seem to be fatal for phantom cosmology, at the quantum level the survival of the universe containing a phantom for 13.7 billion years looks surprising.

\section{Acknowledgments}

It is a pleasure to thank Dr. Sebastiano Sonego for stimulating discussions and an anonymous referee for suggestions leading to improvements in the manuscript. This work was supported by the National Science and Engineering Research Council of Canada (NSERC) through a Discovery Grant. 


\section{References}

[1] A.H. Guth, Phys. Rev. D 23, 347 (1981).

[2] A.G. Riess et al., Astron. J. 116, 1009 (1998); S. Perlmutter et al., Nature 391, 51 (1998); A.G. Riess et al., Astron. J. 118, 2668 (1999); S. Perlmutter et al., Astrophys. J. 517, 565 (1999); A.G. Riess et al., Astrophys. J. 536, 62 (2000); A.G. Riess et al., Astrophys. J. 560, 49 (2001).

[3] I. Zlatev, L. Wang and P.J. Steinhardt, Phys. Rev. Lett. 82, 896 (1999); P.J. Steinhardt, L. Wang and I. Zlatev, Phys. Rev. D 59, 123504 (1999); T. Chiba, Phys. Rev. D 60, 083508 (1999); J.-P. Uzan, Phys. Rev. D 59, 123510 (1999); F. Perrotta, C. Baccigalupi and S. Matarrese, Phys. Rev. D 61, 023507 (1999).

[4] R.A. Daly, M.P. Mory and E.J. Guerra, astro-ph/0203113; R.A. Daly and E.J. Guerra, astro-ph/0209503; R.A. Daly, astro-ph/0212107; S. Hannestad and E. Mortsell, Phys. Rev. D 66, 063508 (2002); A. Melchiorri et al., Phys. Rev. D 68, 043509; P. Schuecker et al., Astron. Astrophys. 402, 53 (2003); R.R. Caldwell, Phys. Lett. B 545, 23 (2002); H. Ziaeepour, astro-ph/0002400; astro-ph/0301640; P.H. Frampton and T. Takahashi, Phys. Lett. B 557, 135 (2003); P.H. Frampton, hep-th/0302007; R.R. Caldwell, M. Kamionkowski and N.N. Weinberg, Phys. Rev. Lett. 91, 071301 (2003).

[5] V. Faraoni, Int. J. Mod. Phys. D 11, 471 (2002).

[6] S.M. Carroll et al., astro-ph/0306438; S. Capozziello et al., astro-ph/0303041; S. Nojiri and S.D. Odintsov, Phys. Rev. D 68, 123512 (2003); A.D. Dolgov and M. Kawasaki, Phys. Lett. B 573, 1 (2003); T. Chiba, Phys. Lett. B 575, 1 (2003); R. Dick, gr-qc/0307052; M.E. Soussa and R.P. Woodard, Gen. Rel. Grav. 36, 855 (2004); D.N. Vollick, Phys. Rev. D 68, 063510 (2003); gr-qc/0312041; X.H. Meng and P. Wang, astro-ph/0307354; astro-ph/0308031; E.E. Flanagan, Phys. Rev. Lett. 92, 071101 (2004).

[7] P.K. Townsend and M.N.R. Wohlfarth, Phys. Rev. Lett. 91, 061302 (2003); M.N.R. Wohlfarth, preprint hep-th/0307179; N. Ohta, Phys. Rev. Lett. 91, 061303 (2003).

[8] We use the metric signature,,,-+++ and units in which the speed of light is unity. $G$ is Newton's constant and the other conventions follow Ref. [9].

[9] R.M. Wald, General Relativity (Chicago: Chicago University Press, 1984).

[10] V. Faraoni, Cosmology in Scalar-Tensor Gravity (Dordrecht: Kluwer Academic, 2004).

[11] L. Parker and A. Raval, Phys. Rev. D 60, 063512 (1999); erratum 67, 029901 (2003); L. Parker and A. Raval, Phys. Rev. D 60, 123502 (1999); erratum 67, 029902 (2003); L. Parker and D.A.T. Vanzella, gr-qc/0312108. 
[12] V.K. Onemli and R.P. Woodard, Class. Quant. Grav. 19, 4607 (2002).

[13] N.D. Birrell and P.C.W. Davies, Phys. Rev. D 22, 322 (1980); N.D. Birrell and P.C.W. Davies, Quantum Fields in Curved Space (Cambridge University Press, Cambridge, England, 1980); B.L. Nelson and P. Panangaden, Phys. Rev. D 25, 1019 (1982). u, Phys. Rev. D 32, 1409 (1987); L.H. Ford and D.J. Toms, Phys. Rev. D 25, 1510 (1982); L. Parker and D.J. Toms, Phys. Rev. D 29, 1584 (1985).

[14] S. Sonego and V. Faraoni, Class. Quant. Grav. 10, 1185 (1993); V. Faraoni and S. Sonego, in Proceedings of the 5th Canadian Conference on General Relativity and Relativistic Astrophysics, R.B. Mann and R.G. McLenaghan eds. (World Scientific, Singapore, 1994).

[15] I. Buchbinder, S.D. Odintsov and I. Shapiro, Effective Action in Quantum Gravity (IOP Publishing, Bristol, 1992); L. Parker and D.J. Toms, Phys. Rev. D 32, 1409 (1985).

[16] M. Szydlowski, W. Czaja and A. Krawiec, astro-ph/0401293.

[17] S.M. Carroll, M. Hoffman, and M. Trodden, Phys. Rev. D 68, 023509 (2003).

[18] C. Barcelo and M. Visser 2000, Class. Quant. Grav. 17, 3843.

[19] C.G. Callan Jr., S. Coleman and R. Jackiw, Ann. Phys. (NY) 59, 42 (1970).

[20] E. Gunzig et al., Class. Quant. Grav. 17, 1783 (2000); Phys. Rev. D 63, 067301 (2001).

[21] S. Foster, Class. Quant. Grav. 15, 3485 (1998); S. Blanco et al., Gen. Rel. Grav. 26, 1131 (1994).

[22] S. Bellucci and V. Faraoni, Nucl. Phys. B 640, 453 (2002).

[23] E. Calzetta and C. El Hasi, Class. Quant. Grav. 10, 1825 (1993); A, Helmi and H. Vucetich, Phys. Lett. A 230, 153 (1997); L. Bombelli et al., J. Math. Phys. 39, 6040 (1998); A.E. Motter and P.S. Letelier, Phys. Rev. D 65, 068502 (2002).

[24] T.M. Rocha Filho et al., Int. J. Theor. Phys. 39, 1933 (2000).

[25] M.A. Castagnino, H. Giacomini and L. Lara, Phys. Rev. D 61, 107302 (2000).

[26] M. Morikawa, Astrophys. J. (Lett.) 362, L37 (1990); Astrophys. J. 369, 20 (1991).

[27] T. Fukuyama et al., Int. J. Mod. Phys. D 6, 69 (1997).

[28] M. Lakshamanan and R. Sahadevan, Phys. Rep. 224, 1 (1993).

[29] L.R. Abramo et al., Phys. Rev. D 67, 027301 (2003). 
[30] Beware of a typo in Ref. [25]: the authors define $x \equiv m \phi$, which should instead read $x \equiv \operatorname{ma\phi }$.

[31] V. Faraoni, E. Gunzig and P. Nardone, Fundam. Cosm. Phys. 20, 121 (1999).

[32] V. Faraoni, submitted for publication (2004).

[33] V. Faraoni, gr-qc/0403020. 